\title{
Simple exercises to flatten your potential
}

\author{
Xi Dong, ${ }^{1,2}$ Bart Horn, ${ }^{1,2}$ Eva Silverstein, ${ }^{1,2}$ and Alexander Westphal ${ }^{3,1}$ \\ ${ }^{1}$ SLAC and Department of Physics, Stanford University, Stanford, California 94305, USA \\ ${ }^{2}$ Kavli Institute for Theoretical Physics, University of California-Santa Barbara, Santa Barbara, California 93106, USA \\ ${ }^{3}$ Deutsches Elektronen-Synchrotron (DESY), Theory Group, D-22603 Hamburg, Germany
}

(Received 22 March 2011; published 27 July 2011)

\begin{abstract}
We show how backreaction of the inflaton potential energy on heavy scalar fields can flatten the inflationary potential, as the heavy fields adjust to their most energetically favorable configuration. This mechanism operates in previous UV-complete examples of axion monodromy inflation-flattening a would-be quadratic potential to one linear in the inflaton field-but occurs more generally, and we illustrate the effect with several examples. Special choices of compactification minimizing backreaction may realize chaotic inflation with a quadratic potential, but we argue that a flatter potential such as powerlaw inflation $V(\phi) \propto \phi^{p}$ with $p<2$ is a more generic option at sufficiently large values of $\phi$.
\end{abstract}

DOI: 10.1103/PhysRevD.84.026011

PACS numbers: $11.25 . \mathrm{Mj}, 98.80 . \mathrm{Cq}$

\section{MOTIVATION: REALIZING YOUR POTENTIAL}

Inflation $[1,2]$ is a powerful framework for addressing the cosmological flatness and horizon puzzles, and for generating the primordial seeds of structure. One recent advance is the development of a model-independent "bottom-up" effective field theory framework [3], which organizes cosmic microwave background (CMB) observables in terms of the lowest dimension operators participating in the effective theory. Still, model building plays an important role, both in field theory ${ }^{1}$ and from the "top down" in string theory. In particular, inflation is sensitive to Planck-suppressed higher dimension operators in the low energy Lagrangian (an infinite sequence of them in the case of large-field inflation with detectable tensor modes). It is therefore of interest to model inflation within a UV-complete candidate for quantum gravity, of which string theory is our best-studied example (see [5] for a recent review).

The extra degrees of freedom of string theory-arising at various mass scales up to the four-dimensional Planck scale-affect the effective action along candidate inflaton directions in field space. This has led to important constraints and complications, such as order-one corrections to slow roll parameters from compactification effects [6] and bounds on the inflationary energy relative to the scale of moduli-stabilizing potential energy barriers $[7,8]$.

Additional fields can play other roles, sometimes in fact contributing useful effects to model building. The string theory motivated possibility of many additional light fields assisting inflation has been addressed in works such as [9], and the tendency of particle production to slow down the inflaton was analyzed in [10]. ${ }^{2}$ In some circumstances, integrating out heavy fields changes the character of the

\footnotetext{
${ }^{1}$ See [4] for a recent example.

${ }^{2}$ For similar approaches using a gas of particles to slow the inflaton field on a steep potential, see e.g. [11,12].
}

inflationary mechanism, producing higher dimension operators suppressed by the inflaton. An early example of this is [13] where off-diagonal Yang-Mills matrix fields renormalize the effective action for the diagonal fields. In [14] similar effects were constructed via integration out of heavy fields coupled through the kinetic term. Integrating out heavy fields can also introduce a field-dependent enhancement of the kinetic term in the inflaton equation of motion [15] or produce features in the power spectrum for small enough radius of curvature in field space (see e.g. [16] for a recent discussion). Effects of heavy fields on precision observables such as the spectral tilt and the tensor to scalar ratio were considered in [17].

In this paper, we show how interactions with heavy scalar fields - such as moduli and Kaluza-Klein (KK) modes - can help flatten the inflaton potential. This mechanism was used in the small-field models of [18] but can occur very generally. The reason is very simple: the heavy fields coupled to the inflaton relax to their most energetically favorable configuration. Consider, as motivation, a simple field theoretic toy model with two fields $\phi_{L}, \phi_{H}$ with the following potential:

$$
V\left(\phi_{L}, \phi_{H}\right)=g^{2} \phi_{L}^{2} \phi_{H}^{2}+m^{2}\left(\phi_{H}-\phi_{0}\right)^{2} .
$$

The light field $\phi_{L}$ will play the role of the inflaton in this toy model. Assuming its kinetic energy is a subdominant effect (as we will shortly confirm), the heavy field will track its instantaneous minimum, which is itself a function of $\phi_{L}$, and so the potential takes the form

$$
V\left(\phi_{L}, \phi_{H, \min }\left(\phi_{L}\right)\right)=\frac{g^{2} \phi_{L}^{2}}{g^{2} \phi_{L}^{2}+m^{2}} m^{2} \phi_{0}^{2} .
$$

For $\phi_{L} \gg m / g$, the inflationary potential is nearly flat. The Friedmann equation becomes $3 H^{2} M_{P}^{2} \approx m^{2} \phi_{0}^{2}$, and

$$
H^{2} \sim m^{2} \frac{\phi_{0}^{2}}{M_{P}^{2}}
$$


We take $\phi_{0}$ to satisfy $0<\phi_{0} \ll M_{P}$ so that $m \gg H$, enforcing that $\phi_{H}$ be heavy enough not to produce scalar perturbations during inflation. ${ }^{3}$ As mentioned above, here we ignored the time derivative terms in the $\phi_{H}$ equation of motion. The ratio between $3 H \dot{\phi}_{H}$ and a typical term $\sim g^{2} \phi_{H} \phi_{L}^{2}$ in $\partial_{\phi_{H}} V$ is tiny in our solution, of order $\left(m / g \phi_{L}\right)^{4}\left(\phi_{0} / \phi_{L}\right)^{2}$.

This mechanism can operate purely within field theory. However string theory naturally provides a wealth of heavy scalar fields coming from moduli stabilization and from Kaluza-Klein modes which may play the role of $\phi_{H}$, as well as potentially lighter fields such as axions and certain brane positions that may play the role of the light inflaton $\phi_{L}$. In a general compactification we expect couplings between axions, fluxes, and geometry. As long as the moduli are not destabilized in the process ${ }^{4}$, the adjustments of the heavy fields will generically go in the direction of flattening the potential. (For restricted couplings, this can fail; for example, if we shifted $\phi_{0}$ by a term proportional to $\phi_{L}$ in the above example, it becomes quadratic at large field values, and can even steepen to quartic for a finite range of $\phi_{L}$ depending on parameter choices.)

One interesting consequence of this concerns $m^{2} \phi^{2}$ chaotic inflation, a classic model [2]. The couplings in the effective action including the light and heavy fields are analytic, and the scalar potential is generically quadratic around an extremum of the potential. In string theory, a key example of such a quadratic term descends from couplings of the form $|B \wedge F|^{2}$ in the low energy effective action, where $B$ is a two-form potential field which produces an axion upon integration over a two-cycle in the compactification. However, although the potential is quadratic near the origin, the response of the heavy fields generically flattens the potential further out. The models of [20] in which the potential ends up linear in $\phi_{L}$ for $\phi_{L}>M_{P}$ is a particular example of this. The present work aims to provide a more systematic understanding of this theoretical trend. (See $[21,22]$ for an interesting discussion of $m^{2} \phi^{2}$ inflation from flux monodromy developed within an effective field theory framework.)

Observationally, a quadratic potential is still viable, currently sitting at the edge of the $1 \sigma$ exclusion contours, with smaller powers (corresponding to flatter potentials) lying further inside the allowed region [19]. Upcoming measurements [23] are expected to significantly improve the constraints on the tensor-to-scalar ratio and the tilt of the power spectrum. Because of the effects of heavy fields, including the flattening effect we consider here, it would not be surprising if the $m^{2} \phi^{2}$ model gets excluded. Special choices of compactification minimizing backreaction may

\footnotetext{
${ }^{3}$ Such fluctuations from additional light fields are constrained by existing limits on isocurvature fluctuations and nonGaussianities in the CMB [19].

${ }^{4}$ Although this is a more energetically favorable outcome, it requires the fields to go over moduli-stabilizing barriers.
}

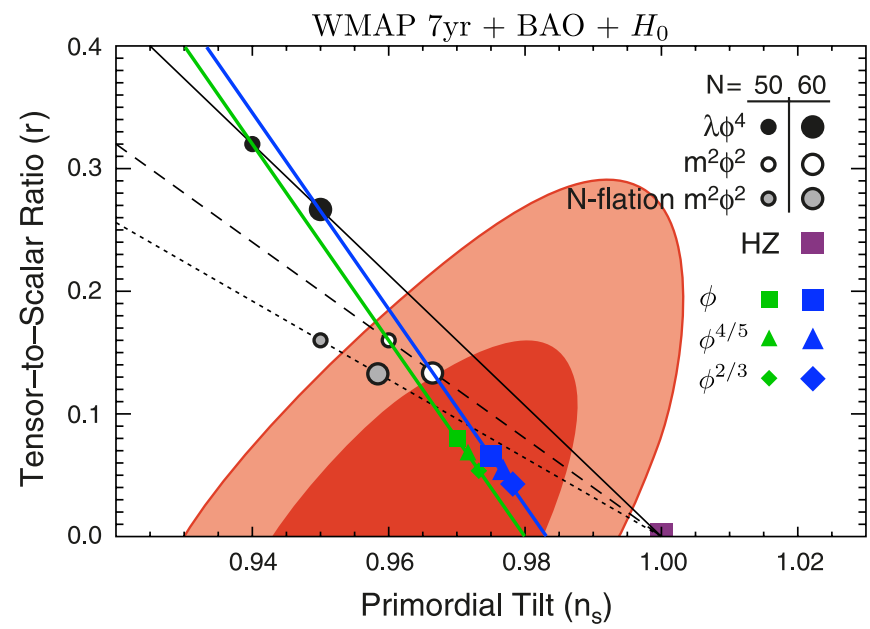

FIG. 1 (color online). Combined data constraints on the tensorto-scalar ratio $r$ and the tilt $n_{s}$ [19] together with the predictions for power-law potentials $\propto \phi^{p}, p>0$ for 50 e-foldings (green line through smaller symbols) and 60 e-foldings (blue line through larger symbols) of inflation. Flattening the potential corresponds to moving down and to the right along these lines. The colored points denote powers that have arisen in various large-field monodromy inflation models in string theory: IIB linear axion monodromy from 5-branes (squares; $\phi$ ), IIA moving 4-brane monodromy (diamonds; $\phi^{2 / 3}$ ), and a candidate example of IIB flux axion monodromy (this work; triangles; $\left.\phi^{4 / 5}\right)$.

realize chaotic inflation with a quadratic potential, but flatter potentials such as power-law inflation $V(\phi) \propto \phi^{p}$ with $p<2$ appear to arise more generically at sufficiently large values of $\phi$. We illustrate the predictions of a flattening monomial power-law potential against the present status of the WMAP 7-year results for the CMB in Fig. 1.

This paper is organized as follows. In the remainder of this section and the next, we introduce the general setup, further specify conditions under which the energetic argument leading to flattening of the potential applies, and describe important situations where it fails. In Sec. III we give several distinct realizations of the effect in the context of axion inflation in string theory, with different fields playing the role of $\phi_{H}$. In Sec. IV we make some concluding remarks.

\section{Additional kinetic effects}

In the toy model presented above, we solved for $\phi_{H}$ in terms of $\phi_{L}$ to good approximation by solving $\partial_{\phi_{H}} V\left(\phi_{L}, \phi_{H}\right) \equiv 0$; the kinetic term $\dot{\phi}_{H}^{2}$ was subdominant. In more general examples we will need to establish whether the same approximation holds. Consider an action (for homogeneous fields) of the form

$$
\int d^{4} x \sqrt{-g}\left\{\dot{\phi}_{H}^{2}+G_{L L}\left(\phi_{H}\right) \dot{\phi}_{L}^{2}-V\left(\phi_{L}, \phi_{H}\right)\right\}
$$


In integrating out $\phi_{H}$, there are two effects that may arise from the kinetic terms. The first, discussed in [15], is that the $\dot{\phi}_{H}^{2}$ kinetic term affects the solution for $\phi_{H}$ as $\phi_{L}$ rolls. This is significant if $\left|d \phi_{H} / d \phi_{L}\right|$ is large compared to $\sqrt{G_{L L}}$. In our examples, as in the above toy model, we will check that this quantity is small.

The second, discussed in [14], arises from the coupling of $\phi_{H}$ in the light field's kinetic term $G_{L L}\left(\phi_{H}\right) \dot{\phi}_{L}^{2}$. If $\dot{\phi}_{L}^{2}$ is large enough during inflation, this term can significantly affect the solution for $\phi_{H}$, leading to a nontrivial k-inflationary [24] effective Lagrangian $\mathcal{L}\left[\left(\partial \phi_{L}\right)^{2}, \phi_{L}\right]$. In this class of models, inflation may occur on a steep potential, with self-interactions of the field $\phi_{L}$ slowing it down (resulting in a large non-Gaussian signature in the power spectrum). The energetics of the backreaction for these more general solutions is not as simple as it is in the limit of slow roll inflation, where the heavy fields adjust in such a way as to flatten the potential when possible. Within slow roll inflation we have $\dot{\phi}_{L}^{2} \ll V$, and this will allow us to self-consistently bound the effect in our examples below. It would be interesting to find UV-complete examples of the effects in $[14,15]$ in future work.

\section{Steepening from kinetic curvature}

We should emphasize that flattening of the potential is not an automatic consequence of couplings to massive fields. For example, even when the kinetic effects of the previous subsection are small it can fail, as can be seen from the following variant of our previous toy model:

$$
\begin{aligned}
\mathcal{L}= & \frac{1}{2} \frac{\phi_{H}}{M_{P}} \dot{\phi}_{L}^{2}+\frac{1}{2} \dot{\phi}_{H}^{2}-g^{2} \phi_{L}^{2} \phi_{H}^{2} \\
& -m^{2}\left(\phi_{H}-\phi_{0}\right)^{2}-\mu^{2} \phi_{L}^{2} .
\end{aligned}
$$

As before, for large $\phi_{L},\left|d \phi_{H} / d \phi_{L}\right|$ is small compared to $\sqrt{G_{L L}}$ and $\dot{\phi}_{H}^{2}$ can safely be neglected, and the kinetic term $\frac{\phi_{H}}{M_{P}} \dot{\phi}_{L}^{2}$ is subdominant to the potential, so that the effects of [14] are suppressed. The canonical field $\tilde{\phi}$ at large $\phi_{L}$ is now $\approx \frac{m}{g} \sqrt{\frac{\phi_{0}}{M_{P}}} \log \left(\phi_{L} / M_{P}\right)$, and the potential has the form

$$
V_{e f f}(\tilde{\phi}) \approx m^{2} \phi_{0}^{2}+\mu^{2} M_{P}^{2} e^{(2 g \tilde{\phi} / m) \sqrt{\frac{M_{P}}{\phi_{0}}}} .
$$

Thus, if $G_{L L}\left(\phi_{H \text {,min }}\left(\phi_{L}\right)\right)$ scales like a negative power of $\phi_{L}$, then the dressed kinetic term is responsible for steepening the potential. This inverse power can arise, for example, in the case where $\phi_{H}$ descends from the overall (inverse) volume of a string compactification, leading to an increased volume at large inflaton field values (fattening the manifold, and steepening the potential). This can be neglected in examples with sufficiently strong volume-stabilizing potential barriers. In a complete example, $\mu$ would likely not be a fixed parameter and all backreaction effects would need to be incorporated consistently.

\section{WARM-UP: REVIEW OF AXION MONODROMY INFLATION}

Our string-theoretic examples grew out of a project aimed at developing the flux version of axion monodromy inflation. Let us begin by briefly reviewing the general discussion of this mechanism in [20]. A flux version of monodromy inflation has been obtained at the level of effective field theory also in [21,22], and the phenomenology of monodromy inflation was further developed in $[25,26]$.

String theory naturally provides axions

$$
b=\int_{\Sigma^{p}} B_{p}, \quad c=\int_{\Sigma^{p}} C_{p}
$$

coming from p-form Neveu-Schwarz-Neveu-Schwarz (NS-NS) and Ramond-Ramond (R-R) fields $B_{p}, C_{p}$ wrapped on p-cycles in the compact directions. Assuming a single scale $L \sqrt{\alpha^{\prime}}$ for the compactification geometry, the canonically normalized field is related to the angular scalar field (7) by

$$
\frac{\phi_{b}}{M_{p}} \sim \frac{b}{L^{p}}, \quad \frac{\phi_{c}}{M_{p}} \sim \frac{g_{s} c}{L^{p}} .
$$

The theory contains couplings between the axions and various fluxes and spacefilling branes that are generically present in compactifications. These couplings introduce monodromy in the axion direction: the system builds up potential energy as $b$ or $c$ traverses its basic period.

In the specific, UV-complete examples discussed in [20] the axion potential is lifted by the Dirac-Born-Infeld (DBI) action

$$
\begin{aligned}
\mathcal{S}_{\mathrm{DBI}} & =-\frac{1}{g_{s} \alpha^{\prime 3}} \int \sqrt{\operatorname{det}\left(G_{M N}+B_{M N}\right) \partial_{\alpha} X^{M} \partial_{\beta} X^{N}} \\
& \Rightarrow V\left(\phi_{b}\right) \propto \sqrt{1+\left(\frac{\phi_{b}}{M_{P}}\right)^{2}}
\end{aligned}
$$

for a spacefilling D5-brane wrapped on the two-cycle (or its $\mathrm{S}$-dual in the case of R-R axions). Using the AdS/CFT correspondence, this result can be described equivalently in terms of a dual geometry plus fluxes. In that description, the monodromy arises from flux couplings of the form

$$
\mathcal{L} \sim\left|B_{2} \wedge F_{3}\right|^{2}+\cdots
$$

or its S-dual $\left|\mathrm{C}_{2} \wedge \mathrm{H}_{3}\right|^{2}$. Although the coupling (10) is quadratic, backreaction of the axion and fluxes on the geometry leads to a linear potential, as we will discuss in more detail below. This provides an explicit example of the general trend discussed in the introduction: that backreaction of the potential energy descending from (10) should flatten the potential, since this is energetically favorable.

Globally, however, the most energetically favorable configuration in metastable string compactifications is the runaway to large radius and/or weak coupling, or decays 
to negative cosmological constant. Therefore, before discussing examples of potential-flattening effects, let us first briefly review the combined conditions for maintaining moduli stabilization and the Cosmic Background Explorer (COBE) normalization of the power spectrum.

As is emphasized in [20], the canonically normalized axion potential is, in the absence of strong warping (supposing for illustration that $B_{2}$ is the inflationary axion),

$$
\frac{1}{\alpha^{\prime 4}} \int d^{6} x \sqrt{-g}\left|B_{2} \wedge F_{q}\right|^{2} \sim \frac{1}{\alpha^{\prime 4}} \frac{\phi_{b}^{2}}{M_{P}^{2}} \int d^{6} x \sqrt{-g}\left|F_{q}\right|^{2} .
$$

If the $q$-form flux lifting the axion potential makes a sufficiently subleading contribution to the moduli stabilization, one can obtain a super-Planckian field range without destabilizing the moduli.

In order to provide a successful phenomenological model of chaotic inflation, we must have a sufficient range to give $N_{e}=60$ e-foldings of inflation, and the power spectrum of scalar perturbations must match the COBE normalization,

$$
\Delta_{\text {scalar }}^{2}=\frac{H^{4}}{(2 \pi)^{2} \dot{\phi}^{2}} \cong 10^{-9} .
$$

For a power-law potential $V(\phi) \propto \mu^{4-n} \phi^{n}$, the required field range is $\Delta \phi / M_{p} \sim \sqrt{n N_{e}}$, which is $O(15)$ for the quadratic case. The COBE normalization becomes

$$
\left(\frac{\mu}{M_{P}}\right)^{2-(n / 2)}\left(\frac{\Delta \phi}{M_{P}}\right)^{(n / 2)+1} \sim 10^{-5},
$$

which becomes $\mu / M_{P} \sim 10^{-6}$ for a quadratic potential and $O\left(10^{-3}\right)$ for a linear potential.

Let us first review the basic scales in the problem which show that it is possible for axion monodromy inflation to self-consistently satisfy the required number of e-foldings and COBE normalization. Here is an estimate of the effects of these observational constraints in the extreme case of $m^{2} \phi^{2}$ inflation, in the absence of warping (flatter potentials and warped models being easier to embed below the moduli-stabilizing barrier, this is the most conservative estimate we can make). Supposing that the inflaton comes from a $C_{p}$ axion lifted by the term $\left|C_{p} \wedge H_{3}\right|^{2}$, the flux potential is

$$
\begin{aligned}
\mathcal{U}= & \frac{1}{\alpha^{\prime 4}} \int d^{6} x \sqrt{-g}\left|C_{p} \wedge H_{3}\right|^{2} \sim \frac{M_{P}^{2}}{\alpha^{\prime}}\left(\frac{g_{s} c}{L^{p}}\right)^{2}\left(\frac{K}{L^{3}}\right)^{2} \\
& \sim M_{P}^{4}\left(\frac{g_{s}^{2} K^{2}}{L^{12}}\right) \frac{\phi_{c}^{2}}{M_{P}^{2}},
\end{aligned}
$$

where we have labeled the number of $H_{3}$ flux quanta by $K$. The condition for realizing 60 e-foldings of inflation without destabilizing the moduli and for matching the power spectrum to the COBE normalization then becomes (returning to the general case of $q$-form flux lifting the inflaton $\sim\left|C_{p} \wedge F_{q}\right|^{2}$ )

$$
15 K_{\mathrm{inf}} \ll K_{\text {moduli }} ; \quad \frac{\mu}{M_{p}} \cong \frac{g_{s} K_{\text {inf }}}{L^{q+3}}(2 \pi)^{7 / 2} \cong 10^{-6} .
$$

These conditions can be satisfied for reasonable parameter values, e.g. $g_{s} \sim 0.02, K_{\mathrm{inf}} \sim 1, q=3, L \sim 10$. Moreover, as already mentioned, warping can naturally suppress the potential energy if the inflationary sector is localized in a region of large gravitational redshift, as in the specific examples in [20]. Therefore there is no immediate obstruction to fitting the flux-based version of axion monodromy inflation into stabilized string compactifications, avoiding catastrophic decay of the vacuum.

More generally, there may be single-sector models where the inflaton potential itself helps stabilize the moduli during inflation, competing with or even dominating over some of the terms in the moduli potential. The gravity dual of the models [20] is a familiar local example of this, where down the brane throat the axion $c=\int_{\Sigma_{2}} C_{2}$ helps stabilize the cycle $\Sigma_{2}$ it threads. Below we will explore potential generalizations of this which are further from a simple brane construction.

\section{Flattening vs moduli potential barriers}

Before proceeding to our main flattening exercises, it is worth describing a simple example which illustrates both the flattening effect and how the requirement of moduli stabilization can cut it off. De Sitter vacua can plausibly be achieved in string theory via perturbative techniques, where localized sources of energy such as curvature, D-branes and NS5-branes, fluxes, orientifolds, and others contribute to an effective potential for the four-dimensional scalar fields, which is minimized to solve the equations of motion. Such constructions were introduced in [27] and discussed in [28]; worked examples include [29-32]. It is useful to organize these mechanisms in terms of an "abc" structure for the potential,

$$
V(g)=a g^{2}-b g^{3}+c g^{4},
$$

where $g$ is a representative modulus such as the string coupling (with the coefficients $a, b$, and $c$ depending on the other moduli). Such a potential generally arises with curvature, Neveu-Schwarz-Neveu-Schwarz fluxes, and/or supercritical dimensionality in the $a$ term, orientifold planes in the $b$ term, and Ramond-Ramond fluxes in the $c$ term. This potential has a positive metastable minimum when the quantity $4 a c / b^{2}$ is minimized as a function of the other moduli, within the window

$$
1<\frac{4 a c}{b^{2}}<\frac{9}{8} .
$$

Adding flux energy from an axion term will produce an effective potential of the form

$$
V(g, x)=a g^{2}-b g^{3}+\left(1+x^{2}\right) c g^{4},
$$




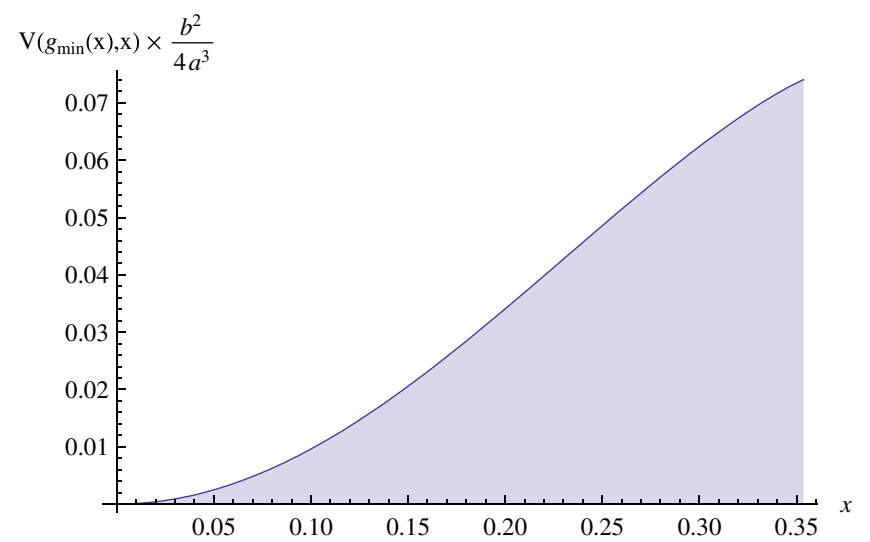

FIG. 2 (color online). Effects of an inflationary flux on the three-term structure stabilized in a Minkowski minimum for $x=0$.

where $x$ is proportional to the axion field. Explicit examples may be found among the axions in [30-32], though we have not developed complete models.

Setting $4 a c / b^{2}=1$, the potential is stabilized at a Minkowski minimum for $x=0$, and as $x$ is turned on, the de Sitter minimum persists as long as

$$
x^{2}<\frac{1}{8}
$$

Including backreaction, $V\left(g_{\min }(x), x\right)$ is no longer quadratic, as plotted in Fig. 2.

As expected, the potential is quadratic for small values of $x$ where backreaction can be ignored, and then flattens as $x$ increases. However, the flattening only starts to become significant when $x$ is of order one, but from (19) it is clear that $x$ begins to destabilize the minimum at this point.

\section{WORKOUT: AXIONS PUSHING ON HEAVY FIELDS}

Finally let us turn to the effects of interest in this paper, the backreaction of the energy (11) and (14) on heavy fields and its effect on the inflationary potential energy.

\section{A. Bowflux: Sloshing of flux on fixed cycles}

The axion potential may be modified by rearrangement of fluxes on fixed cycles so as to minimize their energy. To illustrate this effect, we consider a model of the kind discussed by [33] stabilized by three-form fluxes $H_{3}, F_{3}$. We add a small extra three-form flux $\Delta H_{3}$ to an unwrapped cycle, and turn on an axion $C_{2}$ threading a cycle $\Sigma_{2}$ as the inflaton. Our candidate inflaton will be $c \sim \int_{\Sigma_{2}} C_{2}$. We minimize the other fields at a given value of $c$, given consistency with moduli stabilization which requires that the inflationary energy stay below the moduli-stabilizing barriers. In general the potential will descend from terms in the 10-dimensional action of the form

$$
\frac{1}{g_{s}^{2}}\left|H_{3}+\Delta H_{3}\right|^{2}+\left|C_{2} \wedge\left(H_{3}+\Delta H_{3}\right)\right|^{2}+\left|F_{3}\right|^{2} .
$$

The number of flux quanta threading a given cycle is topological and does not change, but the fluxes may slosh around on their cycles so as to minimize the total energy. If the flux $\Delta \mathrm{H}_{3}$ shifts so that its support is partially separated from that of $C_{2}$, for instance, the Chern-Simons term would be weakened, but the contribution to the potential from the $\left|\Delta H_{3}\right|^{2}$ term would increase. The competition between them determines the optimal field configuration. In general the geometry and the axion wave function can adjust as well. Before considering the potential energy, $C_{2}$ minimizes its energy by forming a flat connection $c \omega_{2}$ (where $\omega_{2}$ is a nontrivial closed form which integrates to one over $\Sigma_{2}$ ). In the presence of potential energy, it might prove energetically favorable for Kaluza-Klein modes of $C_{2}$ to turn on to reduce the second term in (20), at the cost of introducing a contribution to the $\left|F_{3}\right|^{2}$ term. However, to illustrate our effect, let us focus on the sloshing of $\Delta H_{3}$ at fixed $C_{2}$, since the adjustment of any other modes (such as the geometry and $C_{2}$ itself) can only enhance the flattening effect.

Keeping fixed the integral of $\Delta H_{3}$ over the three-cycles it threads, $\Delta H_{3}$ can scrunch up in three directions $w$ along the three-cycle to reduce its overlap with $C_{2}$. Let us denote by $\tilde{L} \sqrt{\alpha^{\prime}}$ the size of the region over which the scrunchedup field $\Delta H_{3}$ has support, modeling its profile locally by

$$
\sqrt{\alpha^{\prime}} \Delta H_{3} \sim \frac{\Delta N}{\tilde{L}^{3}} e^{-w^{2} / \tilde{L}^{2} \alpha^{\prime}},
$$

where $\Delta N$ is the number of $\Delta H_{3}$ flux quanta. We would like to minimize the potential energy with respect to $\tilde{L}$ and determine the effect of this on the axion potential. If the profile of $C_{2}$ were flat in the internal dimensions, shrinking $\tilde{L}$ would not be advantageous. Of course harmonic forms in nontrivial compactification manifolds are not constant. Taylor expanding (and assuming rough isotropy locally), let us model $\mathrm{C}_{2}$ in the region of support of $\Delta \mathrm{H}_{3}$ as

$$
C_{2}(w) \sim \frac{c}{L^{2}}\left(1+\gamma \frac{w^{2}}{L^{2} \alpha^{\prime}}+\cdots\right),
$$

where $\gamma$ is a constant derived from the Taylor expansion of $C_{2}$ 's profile. ${ }^{5}$ Here we are assuming $\Delta H_{3}$ is centered on a local minimum of $C_{2}$, which is its preferred configuration if available [otherwise one would obtain a linear term in the expansion (22), with similar results].

After integrating over the internal volume, the relevant terms in the potential are proportional to

$$
\frac{1}{g_{s}^{2}}\left[\Delta N^{2}\left(\frac{L}{\tilde{L}}\right)^{3}+\gamma N \Delta N\left(\frac{\tilde{L}}{L}\right)^{2} \frac{\phi_{c}^{2}}{M_{P}^{2}}\right]
$$

\footnotetext{
${ }^{5}$ Note that $\mathrm{C}_{2} \wedge H_{3}$ will in general include angular factors depending on the geometry. We will not write these factors here.
} 
where $N$ refers to the number of $H_{3}$ flux quanta and $L \sqrt{\alpha^{\prime}}$ is a typical length scale in the compactification. Here the first term comes from $\left|\Delta H_{3}\right|^{2}$. The second term comes from $\left(C_{2} \wedge H_{3}\right) \cdot\left(C_{2} \wedge \Delta H_{3}\right)$, and gets its leading contribution from the $w^{2}$ term in (22) convolved with (21). ${ }^{6}$ The potential will then minimize these two terms and be proportional to $\phi_{c}^{6 / 5}$, which is flatter than quadratic.

This illustrates the flattening mechanism, but only provides a lower bound on the effect. Adjustments of other fields including $C_{2}$ and the compactification geometry would further flatten the potential.

\section{Bounding additional kinetic effects}

As discussed above in the second part of Sec. I, "Additional kinetic effects," we must check whether it is a good approximation to determine the heavy field $\phi_{H}$ (in this case corresponding to KK modes of $B_{2}$ ) in terms of $\phi_{L}$ by solving $\partial_{\phi_{H}} V \equiv 0$, neglecting the contributions from the kinetic terms. The kinetic effects of [15] are small if $\left|\partial \phi_{H} / \partial \phi_{L}\right| \ll 1$, i.e. if the KK modes of $B_{2}$ that we consider make a negligible correction to $C_{2}$ 's kinetic term. It is straightforward to see that this may be obtained in the present example, as follows. The kinetic term for $\tilde{L}$ descends from the kinetic term for $\Delta H_{3}$ and is

$$
\mathcal{L}_{\text {kin }} \sim \frac{\Delta N^{2} M_{P}^{2}}{\tilde{L}^{3} L^{3}}(\partial \tilde{L})^{2},
$$

giving the canonically normalized field $\phi_{H}$ as

$$
\phi_{H} \sim \frac{\Delta N M_{P}}{\tilde{L}^{1 / 2} L^{3 / 2}}+\text { const. }
$$

Minimizing the potential (23) with respect to $L^{\prime}$, we get

$$
\frac{L}{\tilde{L}} \sim\left(\frac{\gamma N}{\Delta N}\right)^{1 / 5}\left(\frac{\phi_{c}}{M_{P}}\right)^{2 / 5} .
$$

Combining the above equations and writing $\phi_{H}$ as a function of $\phi_{c}$, we get

$$
\frac{d \phi_{H}}{d \phi_{c}} \sim \frac{\Delta N}{L^{2}}\left(\frac{\gamma N}{\Delta N}\right)^{1 / 10}\left(\frac{M_{P}}{\phi_{c}}\right)^{4 / 5},
$$

which can be much smaller than 1 for a reasonable range of parameters.

\section{B. Puffing on the kinetic term}

In the previous subsection we have considered modification of the effective potential due to backreaction on the potential terms. The backreaction of the inflationary potential on the geometry can also affect the kinetic term, realizing the "running kinetic term" mechanism described in [34].

In a simple situation where the NS-NS or R-R field threads a cycle of size $L \sqrt{\alpha^{\prime}}$ that is the same as the

${ }^{6}$ The other terms are either subleading or do not depend on $\tilde{L}$. typical length scale in the compactification, the canonical normalization of the inflaton field is given in terms of the number of axion windings by

$$
\frac{\phi_{b}}{M_{P}} \sim \frac{b}{L^{2}}, \quad \frac{\phi_{c}}{M_{P}} \sim \frac{g_{s} c}{L^{p}},
$$

respectively, for a two-form NS-NS and for a p-form R-R axion. If instead we consider cases where the p-form field is localized (e.g. in a throat) and is therefore threading a much smaller cycle of size $L^{\prime} \sqrt{\alpha^{\prime}}$, the canonically normalized field becomes

$\frac{\phi_{b}}{M_{P}} \sim \frac{b}{L^{12}} \frac{L^{13}}{L^{3}} \sim \frac{b L^{\prime}}{L^{3}}, \quad \frac{\phi_{c}}{M_{P}} \sim \frac{g_{s} c}{L^{\prime p}} \frac{L^{13}}{L^{3}} \sim \frac{g_{s} c L^{13-p}}{L^{3}}$.

Here we are considering the case that the support of the axion is of order the size $L^{\prime} \sqrt{\alpha^{\prime}}$ in all directions in the compactification (as occurs, for example, in the case that $L^{\prime} \sqrt{\alpha^{\prime}}$ describes the size of an internal cycle localized within a Freund-Rubin throat). Now if the inflationary flux backreacts on the size $L^{\prime} \sqrt{\alpha^{\prime}}$ of the wrapped cycle, $L^{\prime}$ will become a function of the axion and this will alter the relation between $b$ or $c$ and the canonically normalized field. The terms of the form $\mid$ axion $\wedge$ flux $\left.\right|^{2}$ push the geometry to expand. Given this, $L^{\prime}(b)$ will vary as a positive power of $b$ and reduce the power of $\phi_{b}$ in the potential. For example, in the case where the size $L^{\prime} \sqrt{\alpha^{\prime}}$ is mostly supported by $\left|B_{2} \wedge F_{3}\right|^{2}$, we have $L^{14} \propto b$ and therefore $\phi_{b} \propto b^{5 / 4}$. In the case where the inflation arises from a Ramond-Ramond field, we will have $p \leq 3$ for magnetic fluxes in six compact dimensions, and so $L^{\prime}(c)$ will either reduce the power of the potential or leave it unchanged.

\section{Bounding additional kinetic effects}

In this example, we solved for the heavy field $L^{\prime}$ in terms of the light field $\phi_{b}$ (or $\phi_{c}$ ) by minimizing the potential in the $L^{\prime}$ direction. Let us now address the question of additional kinetic effects described in the second part of Sec. I in the context of the present model. Before describing the kinetic interactions of $\phi_{b}$ and $L^{\prime}$, let us note that the overall size $L \sqrt{\alpha^{\prime}}$ of the compactification will not be pushed far in the process given a sufficient hierarchy between the inflationary energy and the moduli-stabilizing barriers.

First, let us check whether $\left|d \phi_{H} / d \phi_{L}\right|$ is small. This requires knowledge of the kinetic term for $\phi_{H}$, i.e. the relation between the canonically normalized field $\phi_{H}$ and the modulus $L^{\prime}$. The kinetic term for $L^{\prime}$ descends from the 10-dimensional Einstein term, and in four-dimensional Einstein frame is given by

$$
\int d^{4} x \sqrt{-g} M_{P}^{2}\left(\frac{L^{\prime}}{L}\right)^{6}\left(\frac{\partial L^{\prime}}{L^{\prime}}\right)^{2}
$$

in the above example. From this, the canonically normalized field $\phi_{H}$ is

$$
\phi_{H} \sim M_{P}\left(\frac{L^{\prime}}{L}\right)^{3}
$$


Now, from the above-mentioned scaling $L^{14} \propto b$, $\phi_{b} \propto b^{5 / 4}$, we obtain $\phi_{H} \propto \phi_{b}^{3 / 5}$ and

$$
\left|\frac{d \phi_{H}}{d \phi_{L}}\right| \propto \phi_{L}^{-2 / 5},
$$

which is $\ll 1$ for sufficiently large $\phi_{b}=\phi_{L}$.

Next, let us check that the kinetic term for $\phi_{L}=\phi_{b}$ does not constitute a significant source for $\phi_{H}$ as considered in [14]. To do this, write $L^{\prime} \equiv L_{0}^{\prime} e^{\sigma^{\prime}(t) / M_{P}}$ (note here $\sigma^{\prime}$ is not the canonically normalized field). The relevant terms in the effective action have the form

$$
\int d^{4} x \sqrt{-g}\left(e^{2 \sigma^{\prime} / M_{p}} \dot{\phi}_{b}^{2}-V\left(\sigma^{\prime}, \phi_{b}\right)\right)
$$

Each term in the potential scales like a power of $L^{\prime} \propto e^{\sigma^{\prime} / M_{P}}$. Varying this action with respect to $\sigma^{\prime}$, the first term is of order $\dot{\phi}_{b}^{2} / M_{P}$, much smaller than the second term which is of order $V / M_{p}$ during inflation. Thus we can self-consistently ignore the effect of [14] here.

\section{Weight lifting: Pushing on moduli}

The fact that the axion $\times$ flux energy pushes on the moduli can lead to a similar but distinct effect from the backreaction on the inflaton kinetic term just discussed. One concrete example of this is simply the one developed in [20], described in terms of its gravity dual. Again, the term $\left|\mathrm{C}_{2} \wedge \mathrm{H}_{3}\right|^{2}$ is quadratic in the axion $c=\int C_{2}$. But the axion builds up effective D3-brane charge, and from that point of view the potential should be linear in $c g_{s}$, which is proportional to the effective number of D3-branes. This works out because the generalized five-form R-R flux $\tilde{F}_{5}=C_{2} \wedge H_{3}+\cdots$ backreacts on the moduli, giving a near horizon internal geometry with size $R \sqrt{\alpha^{\prime}}$ depending on $c$ as

$$
R^{4} \sim g_{s} \tilde{N} \sim g_{s} c \int_{S^{3}} H_{3}
$$

as in standard Freund-Rubin solutions. Folding this into the effective action, we see that it scales like

$$
\begin{aligned}
\mathcal{S} & \sim \frac{1}{\alpha^{\prime 4}} \int d^{10} x \sqrt{-G}\left|\tilde{F}_{5}\right|^{2}+\cdots \\
& \sim \operatorname{Vol}(4 \mathrm{~d}) \frac{\tilde{N}^{2}}{R^{10}} \times R^{6} \sim \frac{\tilde{N}}{g_{s}} \operatorname{Vol}(4 \mathrm{~d})
\end{aligned}
$$

as befits a set of D3-branes [here $\operatorname{Vol}(4 \mathrm{~d})$ is the volume of the world volume swept out by the brane]. A straightforward calculation of the four-dimensional effective potential, derived for general warping in [35,36], allows one to reproduce from the gravity side the corresponding fourdimensional Einstein frame potential energy $V$ descending from the brane throat,

$$
V(c) \sim M_{P}^{4}\left(\frac{g_{s}^{2}}{\operatorname{Vol}}\right)^{2} \frac{\tilde{N}}{g_{s}},
$$

where Vol is the compactification volume in string units. In the specific construction [20], the kinetic term of the axion was dominated by the ultraviolet region of the compactification well outside the brane throat. Therefore, in that example the kinetic backreaction of Sec. III B does not apply, but backreaction on the geometry (specifically, on the internal size $R \sqrt{\alpha^{\prime}}$ ) flattens the potential from quadratic to linear. In this example, the kinetic effects are bounded much as in the second part of Sec. IIIB, "Bounding Additional kinetic effects."

\section{Circuit training: Toward more generic UV-complete examples}

A general string compactification involves multiple backreaction effects that are simultaneously important. We have not fully controlled any such example in this paper, but will note here an interesting candidate. Consider an $S^{3}$ localized down a warped throat. Put $M$ units of R-R $F_{3}$ flux on its dual cycle $\tilde{S}^{3}$. On the $S^{3}$ itself, put zero total units of flux, but introduce a topologically trivial configuration of $h$ units of $H_{3}=d B_{2}$ on one hemisphere (north of the equator, say) and $-h$ units on the other (south of the equator). This will dynamically relax back down to zero, and if the geometry were fixed the $\left|H_{3}\right|^{2}$ term would produce a quadratic potential for the integral $b \equiv \int_{\text {equator }} B_{2}=h$ of $B_{2}$ over the equator of the $S^{3}$. Backreaction, however, will change this significantly. Consider starting the system in a configuration in which each hemisphere times the $\tilde{S}^{3}$ with flux is approximately solving the equations of motion as in $[33,37]$. This constitutes, in effect, a 3-brane throat and an anti-3-brane throat at the bottom of the original throat. One can set this up explicitly in terms of two close-by conifold singularities with flux. A similar construction with metastable fluxes on a noncompact Calabi-Yau geometry is studied in [38].

Each throat carries potential energy of order $\tilde{N} \sim M b(t)$ including the backreaction of Sec. IIIC. Moreover, the kinetic energy of $b$ is subject to backreaction as in Sec. III B. The four-dimensional canonically normalized field $\phi_{b}$ in four-dimensional Einstein frame is given by

$$
\begin{aligned}
& \int d^{4} x \sqrt{-g_{E}}\left(\partial \phi_{b}\right)^{2} \\
& \sim \frac{1}{g_{s}^{2} \alpha^{14}} \int d^{10} x \sqrt{-g_{s t}}\left(\partial B_{2}\right)^{2} \\
& \sim \frac{1}{\alpha^{\prime}} \int d^{4} x \sqrt{-g_{s t}}\left(\frac{R^{6}}{g_{s}^{2}}\right) \frac{(\partial b)^{2}}{R^{4}} \\
& \sim \int d^{4} x \sqrt{-g_{E}} M_{P}^{2}\left(\frac{g_{s}^{2}}{V o l}\right)\left(\frac{R^{2}}{g_{s}^{2}}\right)(\partial b)^{2} \\
& \sim \int d^{4} x \sqrt{-g_{E}}\left(\frac{M_{P}^{2}}{V o l}\right) R^{2}(\partial b)^{2} \\
& \sim \int d^{4} x \sqrt{-g_{E}} \frac{M_{P}^{2}\left(g_{s} M\right)^{1 / 2}}{V o l} b^{1 / 2}(\partial b)^{2}
\end{aligned}
$$


and so $\frac{\phi_{b}}{M_{P}} \sim \frac{\left(g_{s} M\right)^{1 / 4}}{\sqrt{V o l}} b^{5 / 4}$. These two effects, taken together, suggest a potential

$$
V\left(\phi_{b}\right)=\mu^{16 / 5} \phi_{b}^{4 / 5} .
$$

However, in order to obtain a concrete prediction for the evolution of this system, we would require a better understanding of the region between the brane and antibrane throats and full control over all sources of backreaction in all directions in field space. This would be interesting to pursue further.

\section{COOLDOWN}

A quadratic inflaton potential may be the simplest possibility from a bottom-up approach, but interactions with heavier fields typically deform the effective action, flattening the potential in the cases discussed here for a simple energetic reason. This is a basic aspect of the UV sensitivity of inflation, complementary to others much discussed in the recent literature. If the upcoming round of $\mathrm{CMB}$ measurements become consistent with the predictions of $m^{2} \phi^{2}$ chaotic inflation, this would significantly constrain the inflaton's couplings to additional fields, including those much heavier than the inflationary Hubble scale. Conversely, if the mild trend in the data toward flatter potentials sharpens, the considerations of this paper may help explain the results.

In the case of axion monodromy inflation, we have outlined two specific mechanisms for backreaction to flatten the axion potential; in general the fluxes and the geometry will seek out the state of lowest energy consistent with the higher dimensional equations of motion. In general, determining the correct form of the potential seems a complicated task. Complete catalogs of the modes found in compactification geometries, such as [39,40], may be of use in constructing more explicit examples. It would also be interesting to see if these considerations apply to other mechanisms for inflation, including general small-field models and models with more generic kinetic terms where the energetic analysis is somewhat more complicated. $^{7}$

\section{ACKNOWLEDGMENTS}

We thank Gary Horowitz, Hans Jockers, Shamit Kachru, Nemanja Kaloper, Albion Lawrence, Louis LeBlond, Juan Maldacena, Liam McAllister, David Morrison, Matt Roberts, Leonardo Senatore, Gonzalo Torroba, and Sho Yaida for helpful discussions. X.D., B.H., and E. S. are grateful to the KITP and the UC Santa Barbara Department of Physics for an excellent year during which the major portion of this work was completed. A. W. would like to thank the KITP at UC Santa Barbara for their warm hospitality in 2010, where part of this work was completed. The research of X. D., B. H., and E. S. was supported in part by the National Science Foundation under Grant No. PHY0551164, by NSF Grant No. PHY-0244728, and by the DOE under Contract No. DE-AC03-76SF00515. B.H. is also supported by Stanford University. The research of A.W. was supported in part by the Alexander von Humboldt Foundation, by NSF Grant No. PHY-0244728, as well as by the Helmholtz Association of German Research Centres under Grant No. HZ-NG-603.

\footnotetext{
${ }^{7}$ It would also be interesting to study backreaction further in models where a cycle size modulus plays the role of the inflaton such as [41] or [42].
}

[1] A. H. Guth, Phys. Rev. D 23, 347 (1981); A. D. Linde, Phys. Lett. B 108, 389 (1982); A. Albrecht and P.J. Steinhardt, Phys. Rev. Lett. 48, 1220 (1982).

[2] A. D. Linde, Phys. Lett. B 129, 177 (1983).

[3] C. Cheung, P. Creminelli, A. L. Fitzpatrick, J. Kaplan, and L. Senatore, J. High Energy Phys. 03 (2008) 014; L. Senatore and M. Zaldarriaga, arXiv:1009.2093; X. Chen, M.-x. Huang, S. Kachru, and G. Shiu, J. Cosmol. Astropart. Phys. 01 (2007) 002.

[4] D. Baumann and D. Green, J. High Energy Phys. 04 (2011) 071.

[5] D. Baumann and L. McAllister, Annu. Rev. Nucl. Part. Sci. 59, 67 (2009).

[6] S. Kachru, R. Kallosh, A. D. Linde, J. Maldacena, L. McAllister, and S. Trivedi, J. Cosmol. Astropart. Phys. 10 (2003) 013.

[7] R. Kallosh and A. D. Linde, J. High Energy Phys. 12 (2004) 004.
[8] E. Silverstein and A. Westphal, Phys. Rev. D 78, 106003 (2008).

[9] S. Dimopoulos, S. Kachru, J. McGreevy, and J. G. Wacker, J. Cosmol. Astropart. Phys. 08 (2008) 003; R. Easther and L. McAllister, J. Cosmol. Astropart. Phys. 05 (2006) 018.

[10] D. Green, B. Horn, L. Senatore, and E. Silverstein, Phys. Rev. D 80, 063533 (2009); L. Kofman and A. Linde (unpublished).

[11] A. Berera, I. G. Moss, and R. O. Ramos, Rep. Prog. Phys. 72, 026901 (2009); A. Berera and T. W. Kephart, Phys. Rev. Lett. 83, 1084 (1999); M. Bastero-Gil, A. Berera, J. B. Dent, and T. W. Kephart, arXiv:0904.2195.

[12] I. Dymnikova and M. Khlopov, Grav. Cosmol. Suppl. 4, 50 (1998); I. Dymnikova and M. Khlopov, Mod. Phys. Lett. A 15, 2305 (2000); Eur. Phys. J. C 20, 139 (2001).

[13] E. Silverstein and D. Tong, Phys. Rev. D 70, 103505 (2004); M. Alishahiha, E. Silverstein, and D. Tong, Phys. Rev. D 70, 123505 (2004). 
[14] A. J. Tolley and M. Wyman, Phys. Rev. D 81, 043502 (2010).

[15] S. G. Rubin, Pis'ma Zh. Eksp. Teor. Fiz. 74, 275 (2001) [JETP Lett. 74, 247 (2001)].

[16] A. Achucarro, J.-O. Gong, S. Hardeman, G. A. Palma, and S. P. Patil, J. Cosmol. Astropart. Phys. 01 (2011) 030.

[17] N. Bartolo and A. Riotto, Mod. Phys. Lett. A 23, 857 (2008).

[18] E. D. Stewart, Phys. Lett. B 345, 414 (1995); G. Lazarides and C. Panagiotakopoulos, Phys. Rev. D 52, R559 (1995); D. H. Lyth and E. D. Stewart, Phys. Rev. D 54, 7186 (1996).

[19] D. N. Spergel et al. (WMAP Collaboration), Astrophys. J. Suppl. Ser. 148, 175 (2003); H. V. Peiris et al., Astrophys. J. Suppl. Ser. 148, 213 (2003); D. N. Spergel et al. (WMAP Collaboration), Astrophys. J. Suppl. Ser. 170, 377 (2007); E. Komatsu et al., Astrophys. J. Suppl. Ser. 180, 330 (2009); 192, 18 (2011).

[20] L. McAllister, E. Silverstein, and A. Westphal, Phys. Rev. D 82, 046003 (2010).

[21] N. Kaloper and L. Sorbo, Phys. Rev. D 79, 043528 (2009).

[22] N. Kaloper and L. Sorbo, Phys. Rev. Lett. 102, 121301 (2009); N. Kaloper and A. Lawrence (work in progress).

[23] F. R. Bouchet (Planck Collaboration), Mod. Phys. Lett. A 22, 1857 (2007).

[24] C. Armendariz-Picon, T. Damour, and V.F. Mukhanov, Phys. Lett. B 458, 209 (1999).

[25] R. Flauger, L. McAllister, E. Pajer, A. Westphal, and G. Xu, J. Cosmol. Astropart. Phys. 06 (2010) 009.

[26] N. Barnaby and M. Peloso, Phys. Rev. Lett. 106, 181301 (2011).

[27] E. Silverstein, arXiv:hep-th/0405068.

[28] M. P. Hertzberg, S. Kachru, W. Taylor, and M. Tegmark, J. High Energy Phys. 12 (2007) 095; S. S. Haque, G. Shiu, B. Underwood, and T. Van Riet, Phys. Rev. D 79, 086005 (2009); C. Caviezel, P. Koerber, S. Kors, D. Lust, T. Wrase, and M. Zagermann, J. High Energy Phys. 04 (2009) 010; R. Flauger, S. Paban, D. Robbins, and
T. Wrase, Phys. Rev. D 79, 086011 (2009); U.H. Danielsson, S. S. Haque, G. Shiu, and T. Van Riet, J. High Energy Phys. 09 (2009) 114; B. de Carlos, A. Guarino, and J.M. Moreno, J. High Energy Phys. 01 (2010) 012; C. Caviezel, T. Wrase, and M. Zagermann, J. High Energy Phys. 04 (2010) 011; G. Dibitetto, R. Linares, and D. Roest, Phys. Lett. B 688, 96 (2010); T. Wrase and M. Zagermann, Fortschr. Phys. 58, 906 (2010); U.H. Danielsson, P. Koerber, and T. Van Riet, J. High Energy Phys. 05 (2010) 090; D. Andriot, E. Goi, R. Minasian, and M. Petrini, J. High Energy Phys. 05 (2011) 028.

[29] A. Maloney, E. Silverstein, and A. Strominger, arXiv:hepth/0205316.

[30] A. Saltman and E. Silverstein, J. High Energy Phys. 01 (2006) 139.

[31] E. Silverstein, Phys. Rev. D 77, 106006 (2008).

[32] X. Dong, B. Horn, E. Silverstein, and G. Torroba, Classical Quantum Gravity 27, 245020 (2010).

[33] S. B. Giddings, S. Kachru, and J. Polchinski, Phys. Rev. D 66, 106006 (2002); S. Kachru, R. Kallosh, A. Linde, and S. P. Trivedi, Phys. Rev. D 68, 046005 (2003).

[34] K. Nakayama and F. Takahashi, J. Cosmol. Astropart. Phys. 11 (2010) 009.

[35] S. B. Giddings and A. Maharana, Phys. Rev. D 73, 126003 (2006).

[36] M. R. Douglas, J. High Energy Phys. 03 (2010) 071.

[37] I. R. Klebanov and M. J. Strassler, J. High Energy Phys. 08 (2000) 052.

[38] M. Aganagic, C. Beem, J. Seo, and C. Vafa, Nucl. Phys. B789, 382 (2008).

[39] D. Baumann, A. Dymarsky, S. Kachru, I. R. Klebanov, and L. McAllister, Phys. Rev. Lett. 104, 251602 (2010).

[40] D. Baumann, A. Dymarsky, S. Kachru, I. R. Klebanov, and L. McAllister, J. High Energy Phys. 06 (2010) 072.

[41] J. R. Bond, L. Kofman, S. Prokushkin, and P. M. Vaudrevange, Phys. Rev. D 75, 123511 (2007).

[42] M. Cicoli, C. P. Burgess, and F. Quevedo, J. Cosmol. Astropart. Phys. 03 (2009) 013. 